\title{
Planck Reference Sky versus WMAP
}

\author{
Sara Ricciardi* $*$ \\ Lawrence Berkeley National Lab \\ E-mail: sara.ricciardieromal.infn.it
}

The aim of this work is to investigate the limitation and possible improvements of Planck Reference Sky via the comparison with the 3-years WMAP data. We simulate these maps using the current model that includes up to four diffuse Galactic components and the compact extraGalactic components: galaxy clusters, infrared sources and radio sources. We examine the maps calculating the angular power spectra and the spatial correlation for different sky cuts (all sky maps, in and out of the galactic plane). We find discrepancies in the power spectra particularly evident at lower frequencies. Focusing on synchrotron model we identify possible main causes. This analysis is extended also to WMAP polarization maps.

$C M B$ and Physics of the Early Universe

20-22 April 2006

Ischia, Italy

\footnotetext{
${ }^{*}$ Speaker.

in collaboration with the WG2 developpers of the Planck Reference SKy
} 


\section{Aim and Method}

The aim of this work is to investigate the limitations and possible improvements of Planck Reference Sky. The Planck Reference Sky consists of a simple model of the CMB foreground organized into a set of easy to use IDL routines, which can predict the sky brightness and polarization at any sky position and frequency. This set of model represents the state of the foregrounds knowledge acquired from the community. The diffuse galactic components model currently includes 4 components which are calculated by distinct IDL functions: Free-Free, Synchrotron, Dust and eventually Spinning Dust. The compact sources components consist in the superposition of three different templates: galaxy cluster, infrared sources and radio sources. For additional information on Planck Reference Sky visit COSMOS on http://cosmos.lbl.gov. A better knowledge of foregrounds contamination will be the key factor to improve our knowledge about CMB; in fact the foreground contamination is estimated to be the main non-systematic sources of contamination (e.g. N. Ponthieu et al. 2005 (10)). We can't suppress this contamination because we are embedded in these galactic and extragalactic emissions. Hence we need to characterize foregrounds contamination in order to be able to discriminate between such mix of incoming foreground radiations and the CMB background (see De Zotti 2002(4) and references therein). This issue is very critical for polarized maps because of the scarcity of data . In order to create these Planck Reference Sky maps we need to generate the maps for all the components according to Planck Reference Sky at WMAP frequency and co-add these maps. We take into account the beam size of the instrument deconvolving the $C_{l}$ for the window functions supplied by the WMAP team. We didn't take into account the noise of WMAP because for the aim of this work it is fair consider WMAP noiseless between $l=1$ to 500 .

\section{Power Spectra and Morphological Comparison}

The first natural step is testing the power spectra of WMAP data versus the power spectra of Planck Reference Sky (Figure 1). The agreement is poor; it is better outside the galactic plane and better in Temperature than in Polarization. The wiggles are not physical but due to the sky cutting. This good agreement in the $\mathrm{W}$ band suggest that the dust model is close to what WMAP measured at least in Temperature. In Polarization instead it would be appropriate to dedicate a proper analysis at this issue. In fact, at least for the full sky, the dominant component in polarization for the Planck reference sky is always synchrotron radiation, even in W-band (Figure 3 right panel). The scarce agreement at lower band where synchrotron emission is dominant shows clearly that we need to check the synchrotron model very carefully.

Another important test is checking correspondences in morphology. An easy manner to do that is simply to make a scatter plot of WMAP map versus Planck Reference Sky. A good agreement in temperature is observed especially in the Galactic plane (correlation $\sim 0.8$ ) but very poor agreement is found in polarization both in and out of the galactic plane for all the bands (correlation $\sim 10^{-2}$ : $10^{-3}$ ). In the figure 2 we plot the results for the representative $\mathrm{W}$ band, omitting the other bands for the sake of brevity. A good agreement in temperature is observed especially in the Galactic plane but very poor agreement is found in polarization both in and out of the galactic plane for all the bands. 

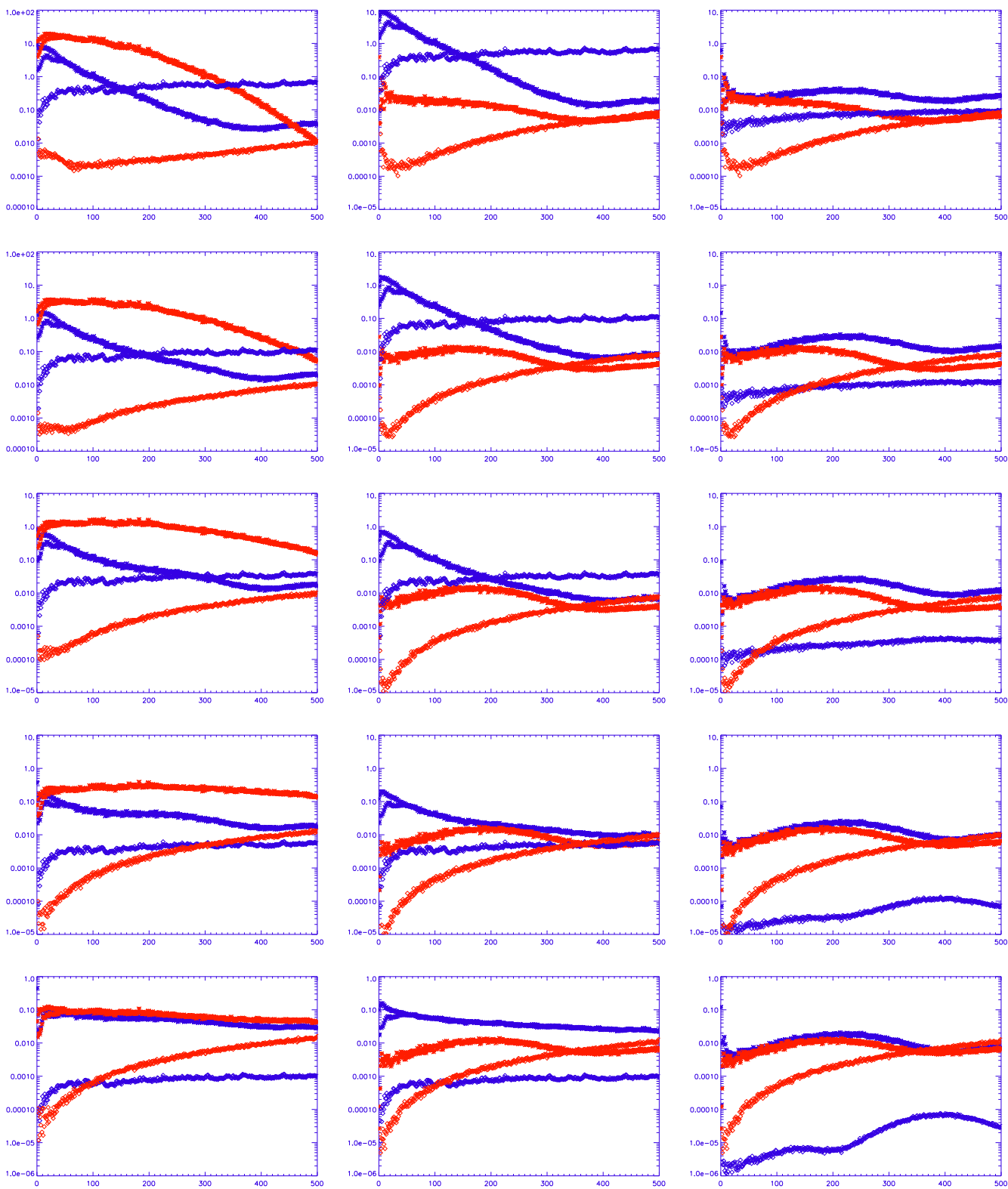

Figure 1: $C l l(l+1) m K^{2}$ of WMAP (red) and Reference Sky (blu) ( $\star$ Temperature and $\diamond$ Polarization), the tree columns are from left to right: all sky, in and out of the galactic plane regions (the cut used is the Kp0 WMAP mask); the rows are the five WMAP bands; top to bottom: K $(23 \mathrm{GHz}) \mathrm{Ka}(33 \mathrm{GHz}) \mathrm{Q}(41 \mathrm{GHz}) \mathrm{V}$ $(61 \mathrm{GHz}) \mathrm{W}(94 \mathrm{GHz})$.

\section{A preliminary comparison with external data set: DRAO survey}

The plot 3 (left panel) shows the polarized power spectra of the following maps: the Planck Reference Sky (Synchrotron Giardino model, Giardino et al. 2002 (6)), the WMAP K band, the DRAO survey (1.4 GHz 47\% sky 36' resolution Wolleben et al. 2006 (12)) and a modified version of the Giardino Model. This plot is very interesting despite the crudeness of the assumption used 

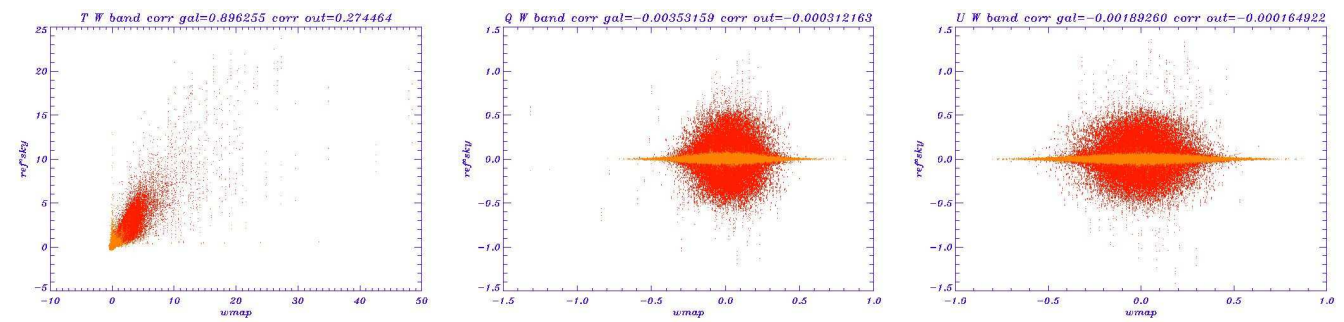

Figure 2: Scatter plots of WMAP versus Planck Reference Sky for the representative W band: in orange the galactic region in red the out galactic region; from the top to the bottom $\mathrm{K}$ to $\mathrm{W}$ (the cut is $\pm 5^{\circ}$; we are going to run this procedure using the Kp0 mask).

to compare so different maps and show how much is urgent to produce a new synchrotron model because even a pure radio survey rigidly rescaled into the cosmological window produce a power spectrum with better agreement with WMAP then the current model. This plot also show that the fraction of polarization is the main responsible for this disagreement. In the following we will explain what we can expect to see in the power spectra comparison and why. In the radio band, Faraday rotation alters the polarization. Electrons in the Galactic magnetic field rotate the plane of polarization because the right and left circular polarization propagate with different velocities in the medium. The rotation is function of the electron density and of the Galactic magnetic field along the line of sight. The net rotation of $\Delta \theta \sim 420^{\circ} / v^{2}$ is negligible in the WMAP bands but it will have a strong effect in radio bands. We rescaled all the maps with a rigid spectral index equal to 3 in the $\mathrm{K}$ band. For this reason we expect to have less power in the power spectrum of DRAO survey rescaled to $\mathrm{K}$ band with respect to the WMAP. We expect the same for the Giardino model that comes from the Haslam map at $0.4 \mathrm{GHz}$ (Haslam et al. 1982 (7)) . Despite the very rough assumption the rescaled DRAO survey is in good agreement with WMAP, and shows slightly less power (as expected). The Giardino Model predicts instead a larger amount of polarization. This evidence, supported also by some observations at 5GHz ( Hummel et al. 1991 (9) ) pushes the fraction of polarization (75\% in Giardino Model) to some lower value (15-20\%). To show how this assumption will affect the amplitude of the power spectrum we plotted a modified version of the Giardino model reducing the polarization fraction from $75 \%$ to $20 \%$. As desirable the power spectrum showed by this modified version of the current model is slightly less then WMAP power spectrum and the agreement with the DRAO survey is very good. In temperature instead the Giardino Model doesn't have enough power especially at medium and small scale ( already after $l \sim 50$ ) because the Haslam map used in the modeling has a very poor resolution.

There are several opportunity to update the Giardino model. All the possible improvement should look at WMAP because this is the only available all sky survey close to the cosmological frequency windows. It will be fundamental to include all possible information coming from new available data set. The knowledge of foreground contamination is essential not just to test the pipeline before the Planck flight but also to improve the performances of blind (Baccigalupi et al. 200020012003 , Delabrouille et al $2003(1 ; 2 ; 3 ; 5)$ ) and non blind component separation algorithms (Hobson et al. 1998 , Stolyarov et al. $2002(8 ; 11)$ ). 

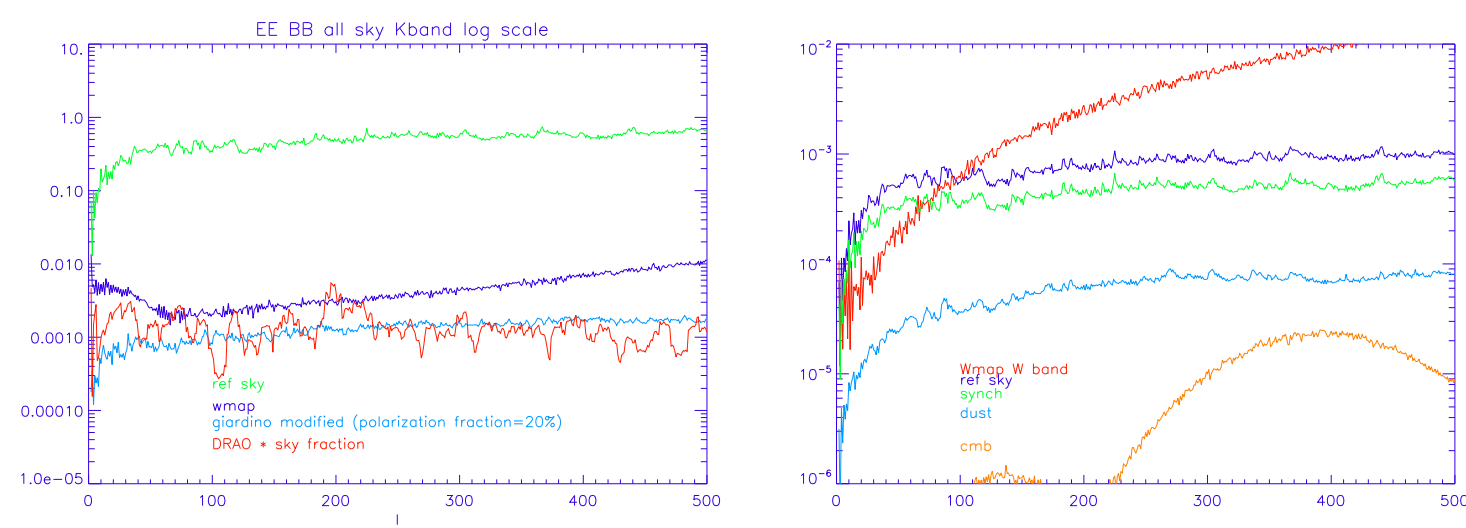

Figure 3: Left panel: $C_{l} l(l+1) m K^{2}$ The Planck Reference Sky (Synchrotron Giardino model) (green), the WMAP K band (blu), the DRAO survey (red) power spectra and a modified version of Giardino Model (light blue) power spectrum (see the text for details) Right panel: the components in polarization for the Planck reference sky in the $\mathrm{W}$ band $C_{l} l(l+1) m K^{2}$.

\section{References}

[1] Baccigalupi C. et al. 2000, MNRAS318, 769

[2] Baccigalupi C. et al. 2001, Astron. \& Astrophys.372, 8

[3] Baccigalupi C. et al. 2003, submitted to MNRAS, preprint astro-ph/0209591

[4] De Zotti G., 2002 in Astrophysical Polarized Backgrounds, AIP conference proc. 609, S. Cecchini, S. Cortiglioni, R. Sault, and C. Sbarra eds., p. 295

[5] Delabrouille J., Cardoso J.F., Patanchon G. 2003, MNRAS346, 1089

[6] Giardino G. et al. 2002, Astron. \& Astrophys.387, 82

[7] Haslam C.G.T. et al. 1982, Astron. \& Astrophys.S 47, 1

[8] Hobson M. et al. 1998, MNRAS300, 1

[9] Hummel, E., et al. 1991, Astron. \& Astrophys., 246, 10

[10] N. Ponthieu et al., Astron. \& Astrophys.444 (2005) 327 [astro-ph/ 0501427 ]

[11] Stolyarov V., Hobson M.P., Ashdown M.A.J., Lasenby, A.N. 2002, MNRAS336, 97

[12] Wolleben, et al., 2006, Astron. \& Astrophys., 448, 411 